\title{
Analysis of condition and risk-based maintenance planning for medium- voltage/low-voltage substations
}

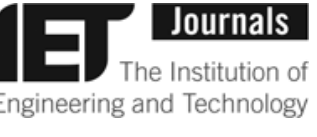

ISSN 2515-0855 doi: 10.1049/oap-cired.2017.0774 www.ietdl.org

\author{
Pascal Köhn ${ }^{1} \bowtie$, Armin Schnettler $^{1}$, Nico Schultze ${ }^{2}$ \\ ${ }^{1}$ RWTH Aachen University, Aachen, Germany \\ ${ }^{2}$ Spie SAG GmbH, Drotmund, Germany \\ $\bowtie$ E-mail: koehn@ifht.rwth-aachen.de
}

\begin{abstract}
Simultaneously increasing cost pressure and the need for grid expansion demands savings in the field of distribution grid operation. The implementation of a risk-based maintenance strategy promises low costs while maintaining high reliability, because equipment is maintained according to its technical condition and importance. Based on a simulative analysis of the influences of medium-voltage grid characteristics on the loss of energy due to failures of the ring main unit, the individual importance of key indicators is determined by the analysis of variance and conjoint analysis. Furthermore, a simulative comparison of condition and risk-based maintenance strategies are performed using a condition-based asset simulation. The results show the impact of the weighting of condition and importance for determining a risk index on the loss of energy and costs for maintenance and fault clearance.
\end{abstract}

\section{Introduction}

The need for savings in the field of grid operation leads to an ongoing implementation of risk-based maintenance schemes to ensure the reliable and safe operation of medium-voltage/ low-voltage (MV/LV) substations. A risk-based maintenance scheme is often used to plan maintenance measures, which bases on the graphically risk assessment shown in Fig. 1. The condition and importance of MV/LV substations are evaluated separately by assessment procedures and plotted on a two-dimensional coordinate system. The risk is determined by calculating the length of the connecting line between the substation's dot and a diagonal intersection of the axis [1].

The weighting of importance and condition can be adapted by varying the angle between the diagonal and the axis. Usually, the angle $\alpha$ is set at $45^{\circ}$ to get equal weights. The actual influence of this angle has not been investigated.

As different condition assessment procedures as well as parameterisations lead to diverse evaluation results, it is necessary to develop methods for the benchmark of assessment procedures for the condition as well as the importance of the equipment and resulting risk.

\section{Condition assessment procedures}

Common condition assessment procedures for MV/LV substations are based on inspection protocols as input. The protocols used comprise 70 items that evaluate the equipment's condition by ratings between 1 and 4 [2]. The interpretation of rating is given in Table 1.

A common assessment procedure is the weighted summation, which evaluates indicators separately and aggregates them to a condition value (see Fig. 2).

For parameterisation of the weighted summation, the analytic hierarchy process (AHP) is used to determine objective weights. The AHP is a mathematical method for decision making that allows accounting for more than one criterion to evaluate different decisions like the importance of indicators. As relevant criteria, the minor and major failure frequency and functional disturbance in the case of malfunction are chosen, because of their influence on the supply reliability. In addition, the repair endurance in the case of malfunction is considered to account for the influence of resupply, after an outage occurred. Furthermore, particular urgency for maintenance and the number of affected customers in case of an outage is taken into account. To ensure objectiveness, the medium rates of minor failures calculated from historic events and the repair endurance is estimated by a distribution system operator. Particularly, critical failures can be considered by knockout rules that lead to the worst possible condition if any critical failures are ascertained [3].

\section{Risk assessment}

If a failure on the MV side of a MV/LV substation occurs, the corresponding MV grid will be disconnected from the feeder by a circuit breaker. The disconnection results in a loss of energy at all $\mathrm{MV} / \mathrm{LV}$ substations of the radial-operated grid. To determine the total loss of energy, the grid's topology, rating of transformers and the availability of remote-controlled short circuit indicators (RCI) have to be considered. The total loss of energy due to substation failures can be used as a measure to determine the individual importance of MV/LV substations.

\subsection{Simulation of energy losses}

MV grids are simulated as radial-operated ring grids. A typical MV grid is shown in Fig. 3. Starting at the busbar of the MV substation, every $\mathrm{MV} / \mathrm{LV}$ substation is looped into the MV grid by a ring main unit with three load disconnectors. If a failure occurs at the ring main unit, the circuit breaker at the corresponding feeder will break the short circuit current and disconnect the stations, as shown in Fig. 3.

The total duration from the failure occurrence until all affected $\mathrm{MV} / \mathrm{LV}$ substations are re-energised must be determined to evaluate the energy loss. Therefore, the failure clearance is simulated, considering the switching of circuit breakers, the analysis of RCIs, the manual search for failures by reading of RCIs inside substations, the re-energisation by switching operations in neighbouring $\mathrm{MV} / \mathrm{LV}$ substations as well as the provision of generators if no switching operation is possible. 
Table 1 Rating of inspection protocol items [2]

\begin{tabular}{lc}
\hline Rating & Interpretation \\
\hline 1 & no visual failure \\
2 & long-term maintenance required/no action required until next \\
& inspection \\
3 & short-term maintenance required \\
4 & immediate maintenance required \\
\hline
\end{tabular}

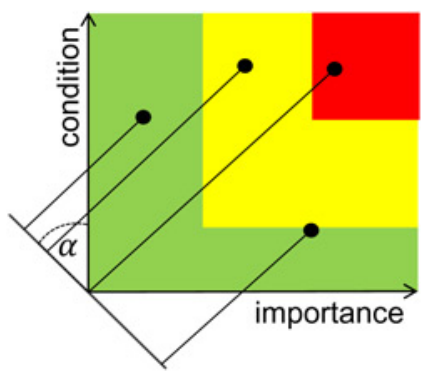

Fig. 1 Graphical risk assessment methodology

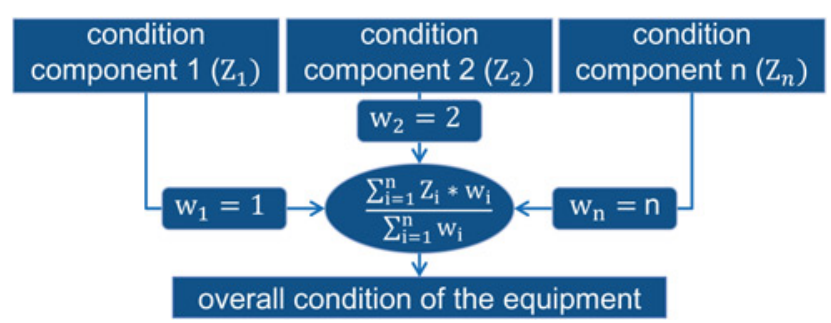

Fig. 2 Overview of the weighted summation [3]

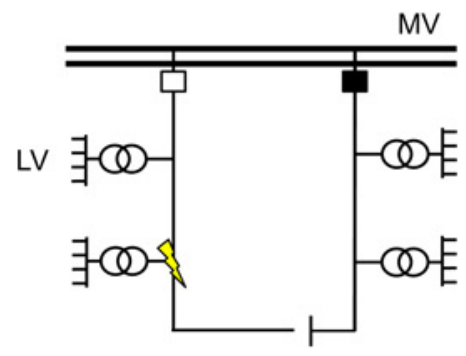

Fig. 3 Typical ring $M V$ grid in radial operation

At first, typical MV grids are generated by varying the number of substations, number of branch lines, number of stations at branch lines, the transformer ratings at the $\mathrm{MV} / \mathrm{LV}$ substations and the availability of RCIs. To vary the number of RCIs, a factor is used that accounts for the relative number of stations in the MV grid with remote-controlled indicators. RCIs are always installed at stations were single feeder start, if available. The value margin of the characteristic numbers is given in Table 2. All possible

Table 2 Margin of characteristic values for the generation of MV grids (radial feeder)

\begin{tabular}{lc}
\hline Characteristic value & Margin \\
\hline number of stations & $4-10$ \\
number of branch lines & $0-2$ \\
station at branch lines & $0-2$ \\
transformer's rating & $50-630 \mathrm{kVA}$ \\
availability factor of RCls & $0-1$ \\
\hline
\end{tabular}

combinations are simulated, except for the transformer rating, which is chosen randomly.

Secondly, a failure at every MV/LV station's ring main unit is simulated, leading the circuit breaker to switch off the radial-operated feeder. Then, possible failure locations are curtailed by analysis of RCIs, if available.

The manual reading of short circuit indicators starts at the first substation behind the MV substation which is not excluded from possible failure locations. If the failed station is arrived, the re-energisation starts by displacement of the open connection point. If substations at branch lines or the failed station cannot be re-energised by switching operations, it is necessary to provide a generator to every station.

It is assumed that it takes roughly $20 \mathrm{~min}$ to arrive and enter a substation and take a reading or to switch a load disconnector [4]. The time to provide and install a generator is assumed to be $90 \mathrm{~min}$ [4]. Furthermore, it is assumed that every transformer is operated at its nominal rating to calculate the loss of energy.

\subsection{Importance and risk assessment}

The importance of characteristics is measured by their impact on the loss of energy. Two different methods, the analysis of variance (ANOVA) and conjoint analysis, are used to determine the importance of each characteristic given in Table 2. Both methods are shortly described for an example of two characteristics $\mathrm{A}$ and $\mathrm{B}$, while detailed information can be found in the literature [5].

All characteristic values are normalised on a scale between 0 and 1 from best to worst expected impact on the loss of energy to eliminate the influence of absolute values. A high availability of RCI for example is expected to have the best influence because the failure location is identified quicker, while a high transformer rating leads to a higher loss of energy during an outage.

The ANOVA determines the effect of characteristics on a target value, in this case the loss of energy. The target value must be of metrical, the characteristics of nominal scale. First the ANOVA divides the total sum of squares of observations of the target value $\mathrm{SS}_{t}$ into sum of squares related to every characteristic $\mathrm{SS}_{x}$, the interaction of $A$ and $B \mathrm{SS}_{A B}$ and to unknown errors $\mathrm{SS}_{w}$ :

$$
\mathrm{SS}_{t}=\mathrm{SS}_{A}+\mathrm{SS}_{B}+\mathrm{SS}_{A B}+\mathrm{SS}_{w}
$$

Every observation $y_{g h k}$ can therefore be determined by the expected value of all observations, the influence of the factors $A\left(\alpha_{g}\right)$ and $B\left(\beta_{h}\right)$, the interaction of $A$ and $B(\alpha \beta)$ and an unknown error at the specific occurrences $g$ of $A$ and $h$ of $B$. The influence is a function of the characteristic's value

$$
y_{g h k}=\mu+\alpha_{g}+\beta_{h}+(\alpha \beta)_{g h}+\varepsilon_{g h k}
$$

where the characteristic's effect $\alpha_{g}$ is the medium difference between the total medium value $\mu$ and the mean value of observations $\overline{y_{h}}$ where the characteristic $A$ takes the value $h$

$$
a_{h}=\overline{y_{h}}-\mu
$$

The characteristic's sum of squares $\mathrm{SS}_{A}$ is divided by the degrees of freedom $\mathrm{df}_{A}$ to determine the variance $\mathrm{MS}_{A}$. Following a $f$-test is used to analyse the influence of the individual characteristic on the loss of energy. The $f$-value $F_{A}$ is calculated and compared to a theoretical value given by the $f$-distribution as follows:

$$
F_{A}=\frac{\mathrm{MS}_{A}}{\mathrm{MS}_{w}}
$$


To measure the effect size of each characteristic, the $\eta^{2}$ value is determined by

$$
\eta_{A}^{2}=\frac{F_{A}-\mathrm{df}_{A}}{F_{A} \mathrm{df}_{A}+\mathrm{df}_{w}}
$$

The conjoint analysis is used as a second method to determine the importance of the characteristics. It is usually used to evaluate the influence of different criteria in decision making of a person based on an ordinal scale. For the application in importance evaluation of MV/LV stations, the subjective choices can be substituted by calculations of the energy loss.

In correspondence to the ANOVA it is assumed that every possible combination $k$ of the $J$ characteristics and their $M$ possible values has an additive influence on the observation

$$
y_{k}=\sum_{j=1}^{J} \sum_{m=1}^{M} \beta_{j m} * x_{j m k}, \begin{cases}x_{j m k}=1 ; & j, m \text { valid for } k \\ x_{j m k}=0 ; & \text { else }\end{cases}
$$

The influences $\beta_{j m}$ are determined so that the result fits the observed value best. A metrical ANOVA without interaction is used to determine the $\beta_{j m}$ for every characteristic's value because all characteristics are metrical scaled

$$
\beta_{j m}=\overline{y_{J m}}-\mu
$$

To determine the relative importance of characteristics, the range of the influence of a characteristic is divided by the sum of all characteristic's ranges

$$
w_{j}=\frac{\max _{m}\left(\beta_{j m}\right)-\min _{m}\left(\beta_{j m}\right)}{\sum_{j=1}^{J}\left(\max _{m}\left(\beta_{j m}\right)-\min _{m}\left(\beta_{j m}\right)\right)}
$$

The weighted summation is used to calculate the importance of MV/ LV substations. While the conjoint analysis determines the relative importance of characteristics directly, the ANOVA measures the individual importance by $\eta_{A}^{2}$. Therefore, $\eta_{A}^{2}$ of all characteristics are normalised so that their sum equals one.

To assess the risk of MV/LV substations, the condition and importance values are plotted on a two-dimensional coordinate system, corresponding to Fig. 1. The risk is evaluated depending on the angle $\alpha$ of the diagonal intersection of the axis. An individual weighting of condition and importance can be achieved by varying $\alpha$ from $0^{\circ}$ (importance) to $90^{\circ}$ (condition).

\section{Condition and risk-based asset simulation}

To analyse the impact of assessment procedures, their parameterisation and the influence of $\alpha$ on the risk assessment, an asset simulation is developed that simulates the development of minor to major faults. As evaluation parameters, the loss of energy and costs of maintenance are determined. It is assumed that substations are inspected in a 4-year period. Minor faults will develop within two inspections. The simulation uses a discrete grade-based system for degradation, in accordance with the inspection protocols. An overview of the simulation steps is shown in Fig. 4.

At first, the population of MV/LV stations is initialised by setting master data parameters. A dataset of stations with different types e.g. compact stations and prefabricated station's is generated in accordance with the population of a German distribution system operator [3]. The station's type affects relevant failures. To allow an importance assessment of the stations, the characteristic values are chosen by random, based on a dataset that contains all generated MV grids according to Table 2. The same selection is used for every simulation in this paper. Every station is modelled considering every item checked by the inspection protocol, besides

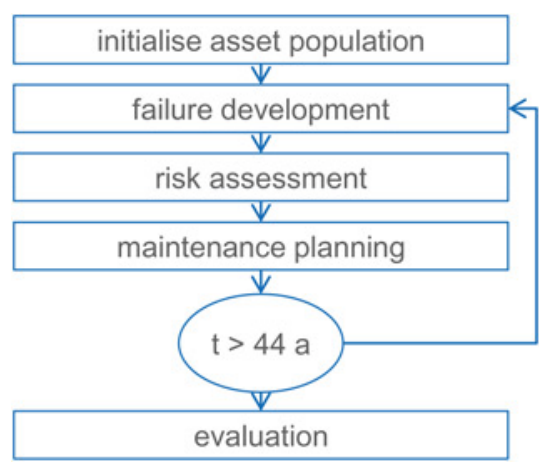

Fig. 4 Overview of the asset simulation [3]

type-specific irrelevant failures. The population consists of 1000 new stations at the beginning of the simulation which are assumed to have their last inspection at an age of 44 years [3].

\subsection{Ageing model for $M V / L V$ stations}

The asset simulation simulates the ageing of a collective MV/LV stations. Every item of the protocol degrades consecutively in discrete states from 1 to 4 , while the degradation from state 4 to 5 is a major failure. For the simulation of discrete states, a Markov chain is used. The transition between two states $i$ and $j$ is represented by a transition probability $p(i, j)$. In this specific case, failures develop within 4 years (see Fig. 5).

Transition rates are calculated using a database of more than 1000 inspection protocols. Possible transitions between the failure states are to remain in the current state or to turn into the next state [3]. The transition probability from state 4 to 5 is chosen to lead to the statistical hazard rate of the individual equipment [6].

\subsection{Simulation of maintenance}

After simulation of degradation, the station's risk values are assessed. Two strategies are considered for maintenance planning. A threshold (TH)-based approach plans to maintain every station whose risk exceeds a given TH. In addition, a budget-based approach is available where stations are maintained in order of their risk as long as the budget is sufficient.

\subsection{Evaluation of simulation results}

To evaluate maintenance strategies, several performance indicators can be considered. The maintenance costs are calculated by summation of material costs, man-hours and the time to arrive to the MV/LV substations. For the calculation of maintenance costs, an appraisal of the failure specific repair time is provided by a distribution system operator and a service provider for maintenance of electrical equipment. The man-hour costs are assumed to be $60 € / \mathrm{h}$ [3]. In addition, the mean time to arrive to the station is assumed to be $20 \mathrm{~min}$ [3]. The component-specific costs of major failures are estimated according to [6]. An example of assumed repair times for some MV equipment is provided in Table 3.

Furthermore, the technical consequences that result from delayed maintenance are evaluated. Therefore, the results of the simulation of energy losses are used to assess the technical effect of faults at the ring main unit. For transformer failures, it is assumed that a power

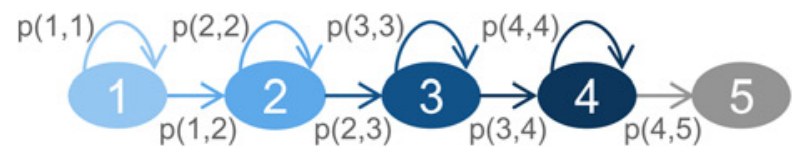

Fig. 5 Markov chain for failure development 
Table 3 Exemplarily repair times of MV components [3]

\begin{tabular}{lccc}
\hline Component & \multicolumn{3}{c}{ Repair time, min } \\
\cline { 2 - 4 } & Rating 2 & Rating 3 & Rating 4 \\
\hline SF6-pressure & 15 & 90 & 300 \\
MV housing & 30 & 60 & 90 \\
MV earthing & 90 & 90 & 90 \\
\hline
\end{tabular}

equal to the transformer's rating is not provided for 90 min until a generator is provided and connected, respectively.

Owing to the stochastic deviation of simulation results due to the probabilistic nature of Markov chains, the simulation is repeated until the mean value of all performance indicators is $<1 \%$ according to the last five iterations.

\section{Simulation results}

Table 4 shows the weights of characteristics used to evaluate the importance of MV/LV substations. The table shows the results of the ANOVA and the conjoint analysis. Most important are the number of stations, the transformer rating and the availability of RCIs.

The number of branch lines and number of substations connected by branch lines is assessed to have a weight of 0.03 or less. While the transformer's rating is evaluated similarly by both methods, the evaluation of the availability of short circuit indicators and number of stations at the MV grid differs.

A simulation of a risk-based maintenance planning strategy shows the impact of different assessment procedures on the total costs (see Fig. 6) and the yearly loss of energy (see Fig. 7). A budget-based maintenance planning approach has been simulated with varying budget for maintenance activities from $€ 0$ to $€ 220,000$. The importance evaluation is parameterised based on the ANOVA and conjoint analysis as well as the transformer's rating only, which is a common procedure in current maintenance practices. $\alpha$ is set to $45^{\circ}$.

It is shown that the use of an assessment procedure that considers the topology of the MV grid and properties of the substation leads to lesser costs and loss of energy at maintenance budgets between $€ 50,000$ and $€ 150,000$. The results also show that if a high or low maintenance budget is provided which leads to numerous or

Table 4 Weights of importance indicators

\begin{tabular}{lcc}
\hline Characteristic value & ANOVA & Conjoint analysis \\
\hline number of stations & 0.75 & 0.40 \\
number of branch lines & 0.03 & 0.00 \\
stations at branch lines & 0.02 & 0.03 \\
transformer's rating & 0.13 & 0.15 \\
availability of RCls & 0.07 & 0.42 \\
\hline
\end{tabular}

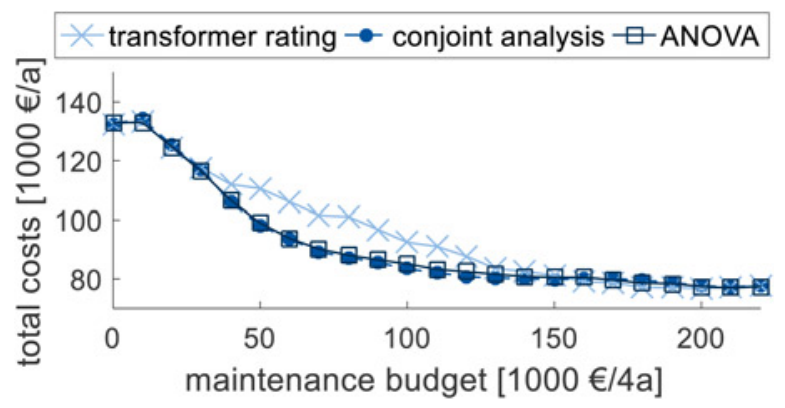

Fig. 6 Influence of different weightings on total costs

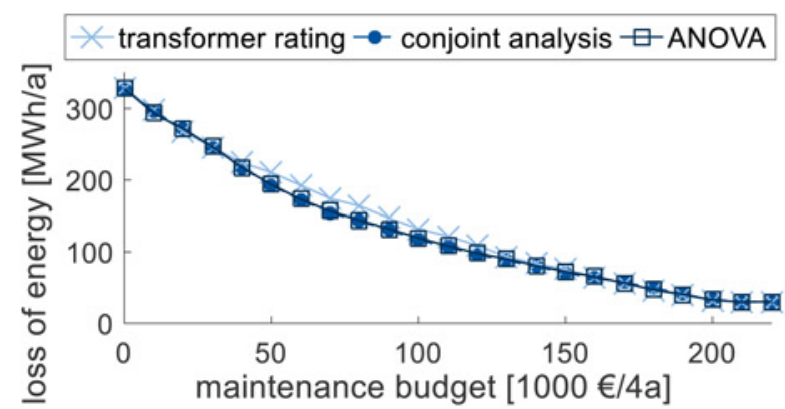

Fig. 7 Influence of weightings on the loss of energy

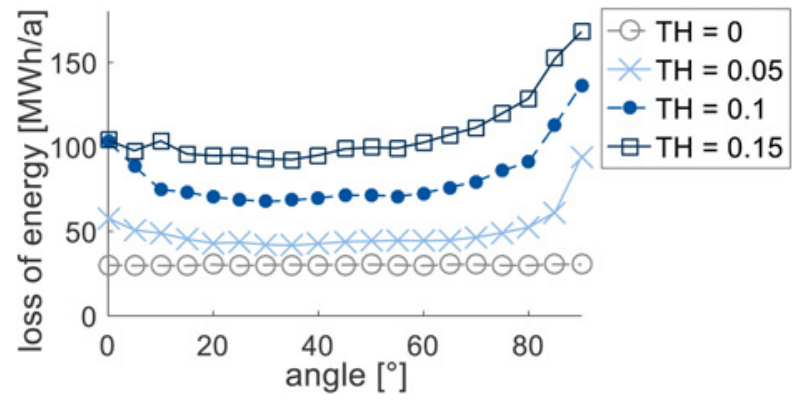

Fig. 8 Influence of $\alpha$ on the loss of energy

rarely maintained substations, the results of different assessment procedures are similar. This agrees with benchmarks of different condition assessment procedures for maintenance planning [3].

Results of an investigation on the effect of the angle for risk assessment on the loss of energy are given in Fig. 8. The ANOVA is used to determine weights for the importance assessment. A TH-based approach is used for maintenance planning where maintenance activities are planned if the risk value exceeds the TH. Four different TH are simulated with $\alpha$ varying from $0^{\circ}$ to $90^{\circ}$.

The results show that the loss of energy can be reduced by considering the station's individual importance. A condition-based strategy is used at angle $90^{\circ}$. The optimal angle is TH dependent between $30^{\circ}$ and $40^{\circ}$. For a TH of 0 which means that every substation is maintained, the angle of the assessment procedure has no influence on the loss of energy.

\section{Conclusion}

Risk-based maintenance schemes promise low maintenance costs while maintaining a high reliability of the grid, because maintenance measures are planned according to the technical condition of the equipment and the consequences in case of failures only. An investigation of the individual loss of energy due to failures at the ring main unit of $\mathrm{MV} / \mathrm{LV}$ substations is undertaken to evaluate the importance of the characteristics of the grid and the station. The results are used to parameterise an importance assessment procedure for MV/LV substations based on the individual influence of these characteristics on the loss of energy as a technical consequence. Therefore, the ANOVA and conjoint analysis are used to determine the individual impact.

Furthermore, the importance assessment procedure is integrated into a risk-based asset simulation that simulates the effect of different maintenance strategies on the costs and loss of energy. The results show that the developed assessment procedures lead to lesser costs and loss of energy than common assessment procedures based only on the transformer's rating. In addition, the influence of the angle for risk assessment of MV/LV substations 
has been investigated. The optimal angle for different risk assessment procedures is determined.

\section{Acknowledgment}

The authors are supported by the Federal Ministry for Economic Affairs and Energy on the basis of a decision by the German Bundestag.

\section{References}

1 Balzer, G., Schorn, C.: 'Asset Management für Infrastrukturanlagen - Energie und Wasser' (Springer-Verlag, Heidelberg, 2011)
2 Johae, C., Beerboom, D., Zdrallek, M., et al.: 'Einsatz geeigneter Messverfahren zur Zustandsbewertung von Mittelspannungs-Ortsnetzstationen' (Diagnostik elektrischer Betriebsmittel, Berlin, 2014)

3 Köhn, P., Schnettler, A., Schultze, N.: 'Simulation and analysis of assessment procedures for condition based maintenance of MV/LV substations'. CIRED Workshop, Helsinki, 2016

4 Forschungsgemeinschaft für Elektrische Anlagen und Stromwirtschaft e.V.: 'Technischer Bericht 302 - Ein Werkzeug zur Optimierung der Störungsbeseitigung für Planung und Betrieb von Mittelspannungsnetzen'. Mannheim, 2008

5 Backhaus, K., Erichson, B., Plinke, W., et al.: 'Multivariate Analysemethoden', vol. 13 (Springer-Verlag, Berlin, 2011)

6 Forschungsgemeinschaft für Elektrische Anlagen und Stromwirtschaft e.V.: 'Entwicklung typspezifischer Prognosemodelle zur Beschreibung der Zuverlässigkeit von Betriebsmitteln im Rahmen des Asset Managements in elektrischen Verteilungsnetzen'. Mannheim, 2013 